\title{
Feasibility of jugular bulb venous monitoring in patients with
} acute ischemic stroke

Ji Man Hong, MD, PhD', Mun Hee Choi, MD', Seong-Joon Lee, MD', Sung Eun Lee, MD', and Jin soo Lee, MD, PhD Department of Neurology, Ajou University Medical Center, Suwon, South Korea'

\section{A)}

\section{Introduction}

- The jugular bulb is the final common pathway for venous blood that drains from the cerebral hemispheres, cerebellum, and brainstem. Jugular bulb venous monitoring can provide information about global cerebral hemodynamics and metabolism. Therefore, it can be an important tool in the clinical management of neurologically injured patients.

- Jugular venous oxygen saturation (Sjo2): Reflects the balance between supply and consumption of oxygen by the brain; Normal SjvO2 values range between $55 \%$ and $75 \%$.: Single episode of desaturation below $50 \%$ is associated with a doubling of the mortality rate.

- Arteriovenous oxygen content (or saturation) difference can be used to relate for changes in metabolism with alterations in CBF. CMRO2 $(\mathrm{VO} 2)=\mathrm{CBF} \times(\mathrm{SaO} 2-\mathrm{Sj}$ O22 $)$

- Lactate has long been viewed as a waste product of oxygen-limited glycolysis and ischemia Lactate has become a key component of neuromonitoring for metabolic crisis through cerebral microdialysis.

- Therefore, we investigated the feasibility and clinical application of jugular bulb venous monitoring in acute ischemic stroke patients at neurocritical care unit.

\section{Methods}

- Patients were recruited from March 2015 to June 20I7. A total of 28 consecutive patients were analyzed with following inclusion criteria: (I) Acute ischemic stroke, (2) NIHSS > 10, (3) Mechanical ventilation with sedation

- Jugular venous catheters were placed in internal jugular vein by ultrasonography-guided method. Lactate, venous oxygen saturation (SjvO2), and arteriovenous oxygen saturation differnece (AVDO2) were measured every 4 hours.

- Metabolic derangement was defined when lactate level was more than $2.0 \mathrm{mmol} / \mathrm{l}$, and venous desaturation group was allocated if $\mathrm{SjvO} 2$ had eve fallen below $60 \%$.

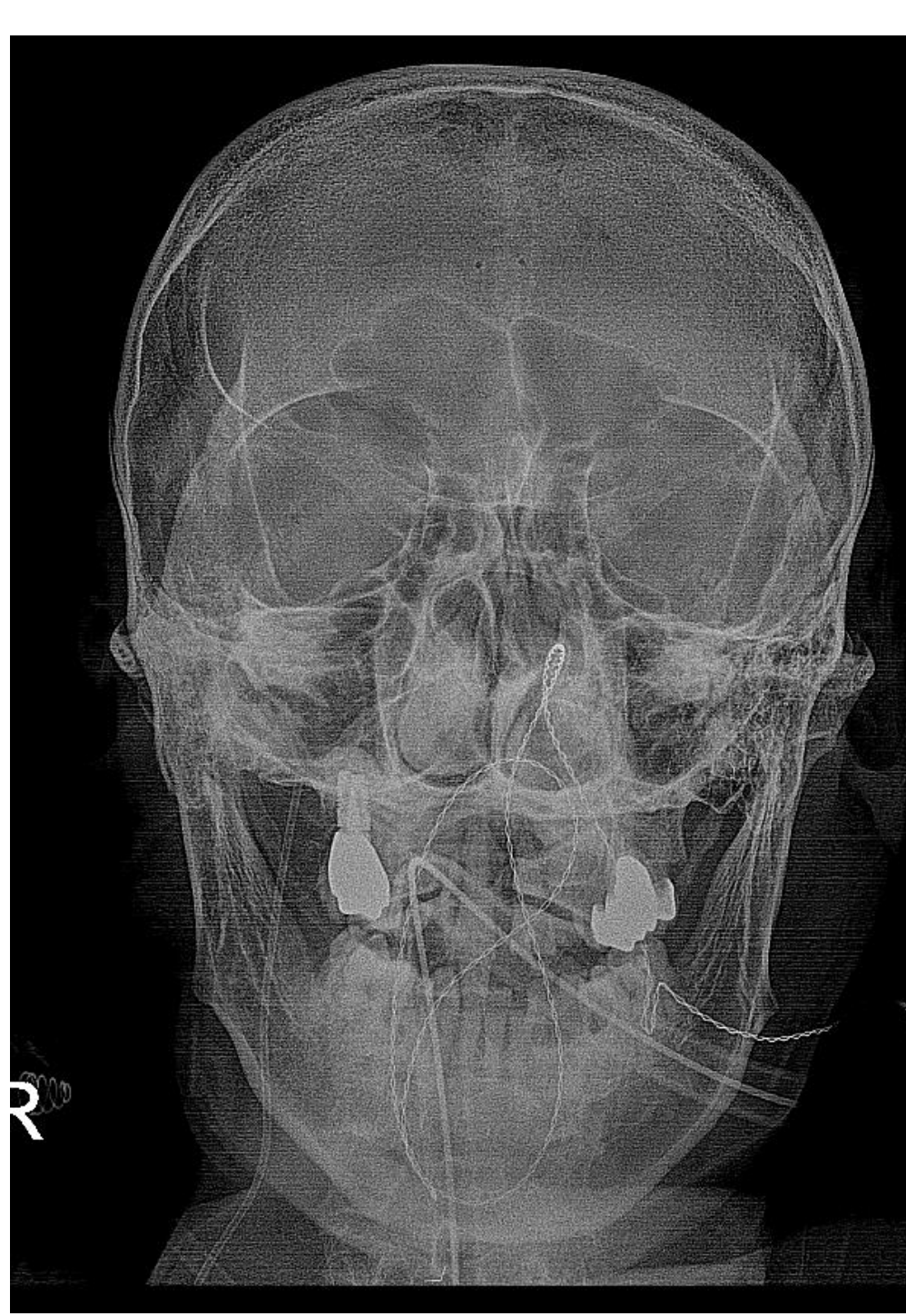

- Patients were divided according to presence of clinical deterioration. Clinical deterioration included neurologic and medical complication after stroke: early neurological deterioration, cerebral edema, requiring surgical intervention, hypotension, cardiac problems (myocardial infarction, arrhythmia), respiratory failure, renal failure. mRS 5-6 at 3 months were defined as poor outcome.

\section{Results}

Table I. Overall patients in this study

$\begin{array}{lr}\text { Overall patients characteristics }(\mathrm{n}=28) & \\ \text { General demographics } & \\ \text { Age, years, mean (range) } & 56.7(33-80) \\ \text { Sex, male, } \mathrm{n}(\%) & 22(78.6) \\ \text { Stroke location, } \mathrm{n}(\%) & \\ \quad \text { Rt MCA } & 10(35.7) \\ \text { Lt MCA } & 14(50.0) \\ \text { Posterior circulation } & 4(14.3) \\ \text { Baseline NIHSS, median (range) } & 20(12-28) \\ \text { Treatment, } \mathrm{n}(\%) & \\ \quad \text { Endovascular recanalization treatment } & 12(42.9) \\ \text { Therapeutic hypothermia } & 15(53.6) \\ \text { Bypass surgery } & 5(17.9) \\ \text { Decompressive craniectomy } & 1(3.6) \\ \text { Metabolic derangement, } \mathrm{n}(\%) & 12(42.9) \\ \text { Venous desaturation, } \mathrm{n}(\%) & 13(46.4) \\ \text { Clinical deterioration, } \mathrm{n}(\%) & 14(50.0) \\ \text { 3mo mRS 5-6, } \mathrm{n} \text { (\%) } & 12(42.9)\end{array}$

- Twelve patients (42.9\%) showed metabolic derangement and experienced mor frequent clinical deteriorations than patients without metabolic derangement $(n=9$, $64.3 \%$ vs. $n=3,21.4 \%, p=0.022$ ).

- Clinical deterioration was noted in 14 patients, and lactate level was significantly higher ( $1.44 \pm 0.48$ vs. I.04 $\pm 0.20 \mathrm{mmol} / \mathrm{l}, \mathrm{p}=0.009$ ).
Table 2. Clinical deterioration

\begin{tabular}{|c|c|c|c|c|c|c|}
\hline & \multicolumn{2}{|c|}{ Clinical deterioration } & \multirow{2}{*}{$P$} & \multicolumn{2}{|c|}{ Estimated OR } & \\
\hline \multirow{2}{*}{\multicolumn{7}{|c|}{ General demographics }} \\
\hline Age, years, mean (range) & $60.3(33-79)$ & & & & & \\
\hline Sex, male, n (\%) & II (78.6) & (1) & 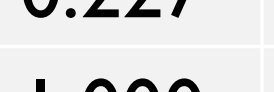 & 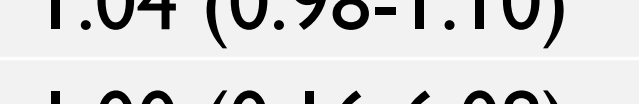 & - & - \\
\hline Stroke location, n (\%) & & (1) & 0387 & & & \\
\hline Rt MCA & $4(28.6)$ & $6(42.9)$ & & (reference) & & \\
\hline Lt MCA & $9(64.3)$ & $5(35.7)$ & & $2.70(0.51-14.37)$ & & \\
\hline Posterior circulation & I (7.I) & $3(21.4)$ & & $0.50(0.04-6.68)$ & & \\
\hline Baseline NIHSS, median (range) & $21(14-26)$ & $18(12-28)$ & 0.511 & $1.06(0.87-1.30)$ & - & - \\
\hline \multicolumn{7}{|l|}{ Jugular bulb monitoring } \\
\hline Mean SvO2, mmHg (SD) & $73.3(5.4)$ & $69.9(6.5)$ & 0.140 & & & \\
\hline AVDO2, $\mathrm{mmHg}(\mathrm{SD})$ & $25.1(5.2)$ & $28.7(6.3)$ & 0.113 & & & \\
\hline Mean lactate, mmol/l (SD) & $1.4(0.5)$ & $1.0(0.2)$ & 0.009 & & & \\
\hline Venous desaturation, n (\%) & $4(28.6)$ & $7(50.0)$ & 0.246 & $0.40(0.08-1.91)$ & - & - \\
\hline Metabolic derangement, $\mathrm{n}(\%)$ & $9(64.3)$ & $3(21.4)$ & 0.022 & $6.60(1.23-35.44)$ & $6.60(1.23-35.44)$ & 0.02 \\
\hline 3mo mRS 5-6, n (\%) & $6(42.9)$ & $6(42.9)$ & 1.000 & & & \\
\hline
\end{tabular}

- Adjusting other potential variables, metabolic derangement was an independent factor associated with clinical deterioration (OR 6.60, 95\% Cl I.23-35.44, $\mathrm{p}=0.028)$. Meanwhile, poor outcome group $(n=12)$ showed no difference on lactate level, but AVDO2 were higher in poor outcome group $(29.54 \pm 5.5 \mathrm{I}$ v. $24.95 \pm 5.65, \mathrm{p}=0.04 \mathrm{I})$. AVDO2 remained an independent factor for poor outcome after adjusting other factors (OR 3.68, 95\% C I.08- $12.55, p=0.038$ ).

Table 3. Clinical outcome

\begin{tabular}{|c|c|c|c|c|c|c|}
\hline & \multirow{2}{*}{\multicolumn{2}{|c|}{$\begin{array}{l}3 \text { mo mRS } \\
\text { Good, 0-4 }(n=16) \text { Poor, } 5-6(n=12)\end{array}$}} & \multirow{2}{*}{ P } & \multicolumn{2}{|c|}{ Estimated OR } & \multirow{2}{*}{$\mathbf{P}$} \\
\hline & & & & crude & adjusted & \\
\hline \multicolumn{7}{|l|}{ General demographics } \\
\hline Age, years, mean (range) & $54.9(33-80)$ & $59.1(36-78)$ & 0.463 & $1.02(0.97-1.08)$ & - & - \\
\hline Sex, male, n (\%) & $14(87.5)$ & $8(66.7)$ & 0.354 & $0.29(0.04-1.92)$ & & \\
\hline Stroke location, $\mathrm{n}(\%)$ & & & 0.124 & & & \\
\hline RtMCA & $8(50.0)$ & $2(16.7)$ & & (reference) & & \\
\hline Lt MCA & $7(43.8)$ & $7(58.3)$ & & $4.00(0.62-25.96)$ & & \\
\hline Posterior circulation & I (6.2) & $3(25.0)$ & & $12.00(0.77-186.36)$ & & \\
\hline $\begin{array}{l}\text { Baseline NIHSS, median } \\
\text { (range) }\end{array}$ & $18.5(14-28)$ & $20(12-24)$ & 0.873 & $0.97(0.79-1.18)$ & - & - \\
\hline Clinical deterioration, $\mathrm{n}(\%)$ & $8(50.0)$ & $6(50.0)$ & 1.000 & $1.00(0.22-4.47)$ & & \\
\hline \multicolumn{7}{|l|}{ Jugular bulb monitoring } \\
\hline Mean SvO2, $\mathrm{mmHg}(\mathrm{SD})$ & $73.2(6.0)$ & $69.4(5.8)$ & 0.098 & $0.88(0.77-1.03)$ & & \\
\hline AVDO2, $\mathrm{mmHg}(\mathrm{SD})$ & $25.0(5.7)$ & $29.5(5.5)$ & 0.041 & $1.17(1.00-1.37)$ & $1.25(1.04-1.51)$ & 0.018 \\
\hline Mean lactate, $\mathrm{mmol} / \mathrm{l}(\mathrm{SD})$ & $1.2(0.5)$ & $1.2(0.3)$ & 0.873 & $0.83(0.13-5.33)$ & - & - \\
\hline Venous desaturation, $\mathrm{n}(\%)$ & $4(25.0)$ & $7(58.3)$ & 0.074 & $4.20(0.84-21.05)$ & & \\
\hline $\begin{array}{l}\text { Metabolic derangement, } \mathrm{n} \\
(\%)\end{array}$ & $7(43.8)$ & $5(41.7)$ & 0.912 & $0.92(0.20-4.18)$ & & \\
\hline
\end{tabular}

- Meanwhile, poor outcome group $(n=12)$ showed no difference on lactate level, but AVDO2 were higher in poor outcome group $(29.54 \pm 5.5 \mathrm{I}$ v. $24.95 \pm 5.65, p=0.04 \mathrm{I})$ AVDO2 remained an independent factor for poor outcome after adjusting other factors (OR 3.68, 95\% Cl I.08-12.55, $p=0.038$ ).

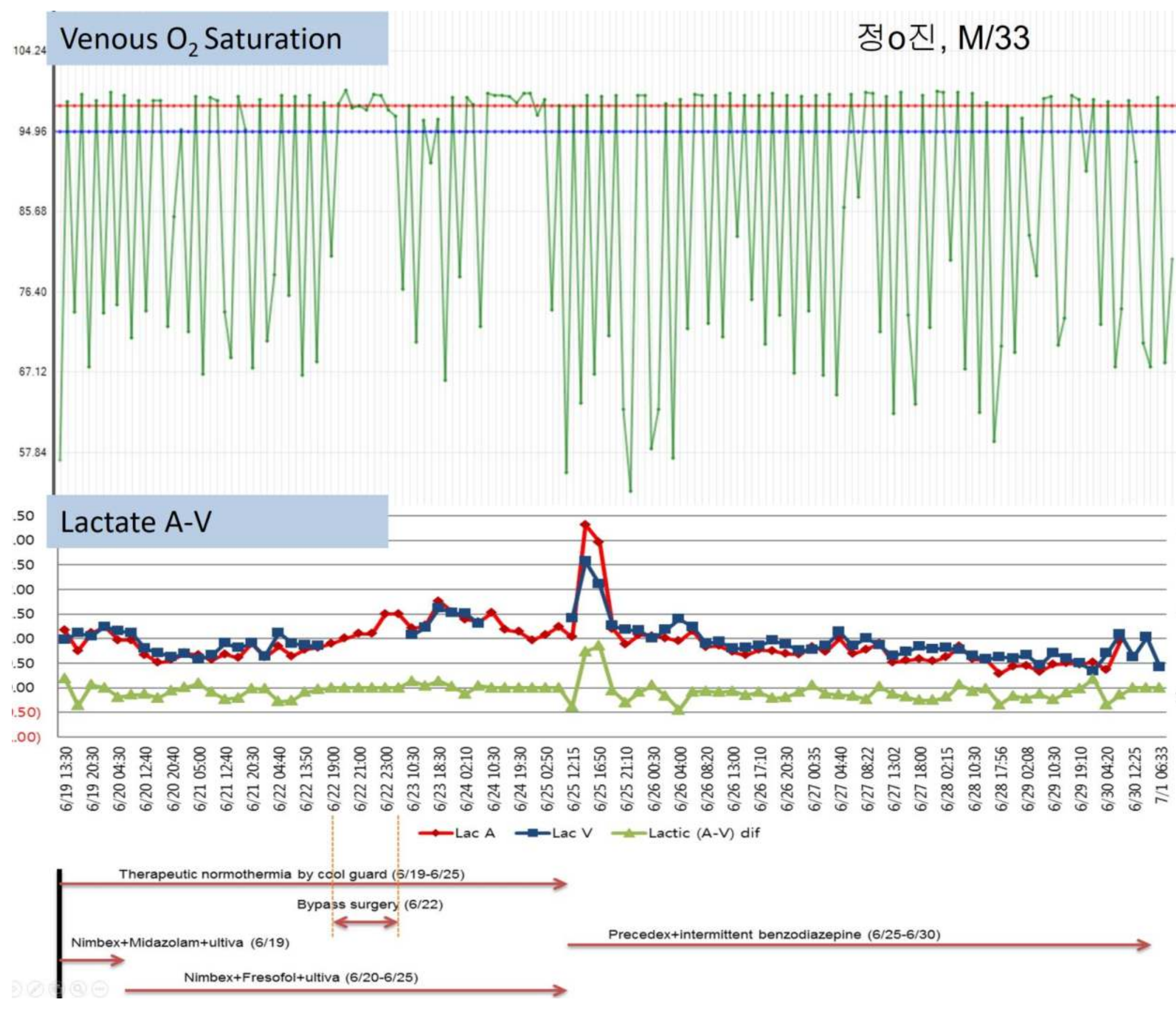

Metabolic derangement pattern

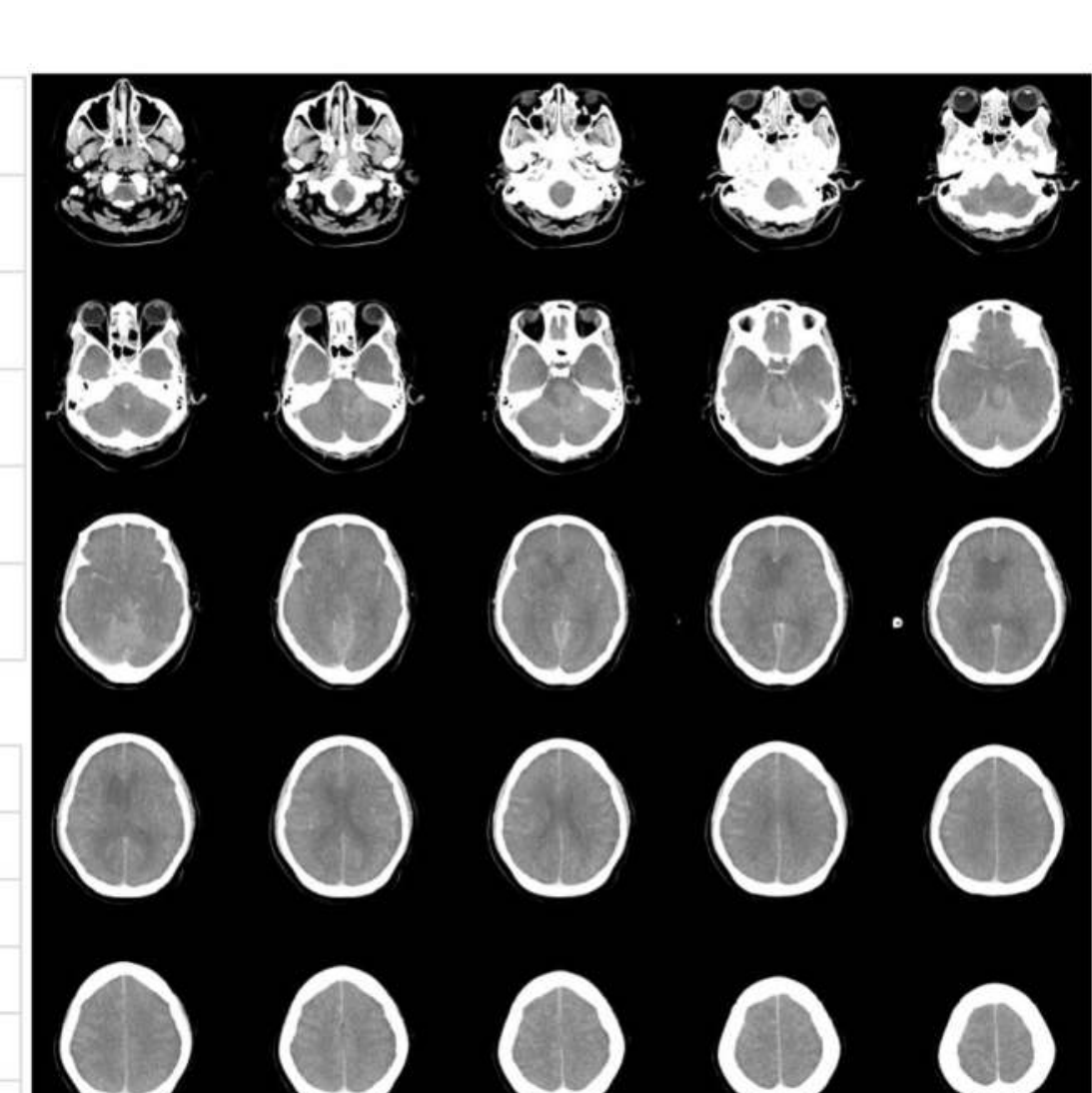

Shunt pattern

\section{Conclusion}

- Jugular bulb venous monitoring is a feasible tool as point-of-care in patients with acute ischemic stroke at neurocritical care unit.

- No complication or adverse events related with the insertion of jugular venous catheter and jugular bulb monitoring (average period $=4.9$ days)

- Lactate elevation was associated with clinical deterioration during neurocritical care. - Venous desaturation contributed to long-term prognosis.

- Limitations: Relative small sample size; Retrospective analysis from a single referral center;Various factors can affect jugular bulb measurements. 